\title{
Inquérito sorológico e fatores de risco para a brucelose por Brucella canis em cães do município de Santana de Parnaíba, Estado de São Paulo ${ }^{1}$
}

\author{
Sérgio S. Azevedo ${ }^{2 *}$, Sílvio A. Vasconcellos ${ }^{3}$, Clebert J. Alves ${ }^{4}$, Lara B. Keid ${ }^{2}$, Lília M.P. \\ S. Grasso ${ }^{5}$, Roberta Mascolli ${ }^{2}$ e Sônia R. Pinheiro ${ }^{3}$
}

\begin{abstract}
Azevedo S.S., Vasconcellos S.A., Alves C.J., Keid L.B., Grasso L.M.P.S., Mascolli R. \& Pinheiro S.R. 2003. [Serological survey and risk factors for brucellosis due to Brucella canis in dogs of the Santana de Parnaíba municipality, State of São Paulo.] Inquérito sorológico e fatores de risco para a brucelose por Brucella canis em cães do Município de Santana de Parnaíba, Estado de São Paulo. Pesquisa Veterinária Brasileira 23(4):156-160. Depto Medicina Veterinária Preventiva, FMVZ/USP, 05508-090 São Paulo, SP, Brazil. E-mail: sevedo@fmvz.usp.br

The prevalence of brucellosis due to Brucella canis was investigated in dogs of the Santana de Parnaíba county, State of São Paulo, southeastern Brazil, and the risk factors for infection were analyzed. For this purpose, 410 blood samples were collected from dogs during the rabies vaccination campaign, in August 1999. The agar gel immunodiffusion test (AGID), using lipopolysaccharides and protein antigens from Brucella ovis, strain Reo 198, was applied first as a screening test on normal sera, and secondly, for confirmation. The same AGID test was applied to sera treated previously with 2-mercaptoethanol (ME-AGID). The complement fixation test (CFT), using B. ovis antigen, strain 63/290, was applied also as a confirmatory test. For the prevalence analysis, animals presenting positive results in both ME-AGID and CFT were considered positive. The prevalence of brucellosis due to $B$. canis was $2.2 \%(95 \%$ C.I. $=1.01-4.13 \%)$. Dogs that were allowed by their owners to stay free outside their home had a higher risk for contracting $B$. canis infection, with an odds ratio value of $8.73(95 \%$ C.I. $=1.48-51.55)$ and $\mathrm{p}=0.04$.
\end{abstract}

INDEX TERMS: Animal brucellosis, Brucella canis, diagnosis, dogs.

RESUMO.- Foi investigada a prevalência da brucelose causada por Brucella canis em cães do município de Santana de Parnaíba, SP, Brasil, e realizado um estudo de possíveis fatores de risco associados à soropositividade para $B$. canis. Foram examinadas 410 amostras de soro sanguíneo de cães colhidas durante a campanha de vacinação anti-rábica ani-

\footnotetext{
${ }^{1}$ Recebido em 25 de setembro de 2002.

Aceito para publicação em 28 de maio de 2003.

${ }^{2}$ Programa de Pós-Graduação em Epidemiologia Experimental e Aplicada às Zoonoses, Depto Medicina Veterinária Preventiva e Saúde Animal, Faculdade de Medicina Veterinária e Zootecnia (FMVZ), Universidade de São Paulo (USP), Av. Prof. Dr. Orlando Marques de Paiva 87, São Paulo, SP 05508-900, Brasil. *Autor para correspondência. E-mail: sevedo@fmvz.usp.br

${ }^{3}$ Depto Medicina Veterinária Preventiva e Saúde Animal, FMVZ/USP, São Paulo, SP.

${ }^{4}$ Depto Medicina Veterinária, Campus VII/Universidade Federal de Campina Grande, Patos, PB 58700-000.

${ }^{5}$ Instituto Biológico, São Paulo, SP.
}

mal, realizada em agosto de 1999. A imunodifusão em gel de ágar (IDGA), utilizando antígeno de lipopolissacarídeos e proteínas de Brucella ovis, amostra Reo 198, foi empregada em soros normais como teste de triagem, e, para a confirmação, a mesma técnica foi aplicada em soros tratados pelo 2-mercaptoetanol (IDGA-ME). A reação de fixação de complemento (CFT), utilizando antígeno de B. ovis, amostra $63 / 290$, também foi utilizada como prova confirmatória. A determinação da prevalência considerou como positivos os animais que reagiram positivamente nos dois testes confirmatórios (IDGA-ME e CFT). A prevalência da $B$. canis foi de $2,2 \%$ (I.C. $95 \%=1,01-4,13 \%$ ). A análise estatística mostrou que os cães com acesso irrestrito à rua o dia todo (manejo do tipo solto) estiveram mais expostos ao risco da infecção por $B$. canis, com um valor de odds ratio de 8,73 (I.C. $95 \%=1,48-51,55)$ e $\mathrm{p}=0,04$.

TERMOS DE INDEXAÇÃO: Brucelose animal, Brucella canis, diagnóstico, cães. 


\section{INTRODUÇÃO}

A brucelose canina por Brucella canis é uma doença infectocontagiosa, de caráter zoonótico, caracterizada, principalmente, por abortamentos e esterilidade nas fêmeas e orquite e epididimite nos machos (Carmichael 1990, Johnson \& Walker 1992, Carmichael \& Greene 1998). Foi descrita, inicialmente, nos Estados Unidos da América, durante episódios de abortamentos em canis de New Jersey (Carmichael 1966).

No Brasil, a presença de cães infectados por $B$. canis foi confirmada tanto por investigações que isolaram o agente (Fernandes et al. 1976/77, Godoy et al. 1977, Larsson 1979, Larsson \& Costa 1980, Vargas et al. 1996 e Gomes et al. 1999), como por inquéritos sorológicos que identificaram a presença de anticorpos séricos contra B. canis (Sandoval et al. 1976, Wald \& Fernandes 1976/77, Godoy et al. 1977, Larsson et al. 1981, Germano et al. 1987, Côrtes et al. 1988, Melo et al. 1997/98, Alves et al. 1999, Maia et al. 1999, Molnar et al. 2001, Moraes et al. 2001 e Moraes et al. 2002).

O caráter zoonótico da brucelose canina por $B$. canis deve ser considerado em face da complexa relação da população canina com os seres humanos (Côrtes et al. 1988) e principalmente pelo estreito contato estabelecido entre cães e crianças. Até o momento há o registro de mais de 35 casos de infecção por $B$. canis em seres humanos em todo o mundo, tanto em infecções naturais como naquelas adquiridas em laboratório como doença ocupacional. $\mathrm{O}$ contato com cadelas que abortaram foi a forma de contágio mais frequiente; no entanto, há alguns relatos de exposição relacionada a cães machos e inclusive alguns casos em que não foi possível a identificação de uma fonte de infecção (Carmichael \& Greene 1998). Os principais sinais clínicos nos seres humanos são febre, calafrios, fadiga muscular, sudorese profusa, mal-estar, linfadenomegalia e perda de peso. As raras complicações incluem endocardite, miocardite, pericardite, meningite, artrite, hepatite e abscessos viscerais (Greene \& George 1984, Hartigan 1997, Carmichael \& Greene 1998).

Roxo et al. (1990), em São Paulo, SP, relataram um caso de brucelose por $B$. canis em um adolescente de 14 anos que apresentou febre, sudorese profusa, forte sensação de cansaço e sintomas psicóticos, com quadro depressivo intercalado com histerismo, confusão mental, rigidez da nuca e priapismo. $\mathrm{A}$ informação da ocorrência de zooerastia levou à suspeita da possível fonte de infecção ter sido uma cadela da raça Doberman, adulta, com quatro anos de idade, positiva à sorologia para $B$. canis.

O município de Santana de Parnaíba situa-se entre as rodovias Castelo Branco e Anhanguera, e serve como corredor de ligação entre as duas estradas. A localização geográfica do município sugere a importância do monitoramento de algumas doenças de caráter zoonótico já registradas, como a leptospirose, e de outras emergentes que poderão vir a ser introduzidas devido à presença constante de fatores predisponentes como trânsito de veículos e proximidade com o ambiente silvestre.

Considerando a importância da brucelose como zoonose, os objetivos do presente trabalho foram determinar a prevalência da brucelose canina por $B$. canis e verificar possíveis fatores de risco para a doença.

\section{MATERIAL E MÉTODOS}

\section{Área de estudo}

$O$ experimento foi conduzido durante a campanha de vacinação anti-rábica animal do município de Santana de Parnaíba, SP, realizada em agosto de 1999. Dados oficiais demonstravam que, em anos anteriores, a cobertura vacinal havia sido de aproximadamente $97 \%$. Esse fato viabilizou a utilização dos postos fixos de vacinação como locais de colheita de sangue dos cães, para a obtenção de uma amostragem que representasse a realidade dos vários bairros do município. Para tanto, a colheita acompanhou o cronograma utilizado na campanha de vacinação, que passa por todos os bairros da cidade.

\section{Amostragem}

A amostra colhida foi calculada com base na população total de cães do município, estimada a partir da população humana de 60.000 habitantes. Para o cálculo da proporção cão/homem foi utilizada a relação de 1:6 (Reichmann et al. 1999), que redundou em um total de 10.000 animais. $O$ cálculo da amostra foi executado com o programa Epi Info 6.0, considerando-se um nível de confiança de $95 \%$, a possibilidade de deteç̧ão da doença de $50 \%$ (correspondente a doenças de desconhecida ocorrência em determinada população) e um erro estatístico de $4,74 \%$, resultando no n amostral de 410 . Em cada posto de vacinação o número de amostras colhidas foi previamente determinado por sorteio probabilístico aleatório de acordo com a ordem cronológica, até perfazer 410 amostras.

\section{Provas sorológicas}

Imunodifusão em gel de ágar (IDGA). Foram utilizados "kits" produzidos pelo Instituto de Tecnologia do Paraná (TECPAR). A técnica foi executada de acordo com as recomendações do fabricante, utilizando-se antígeno de lipopolissacarídeos e proteínas de Brucella ovis, amostra Reo 198. Para confirmação, a mesma técnica foi aplicada em soros tratados pelo 2-mercaptoetanol (IDGA-ME), conforme descrito por Keid (2001).

Reação de fixação de complemento (CFT). Foi executada conforme descrito por Garin-Bastuji \& Blasco (1996), utilizando-se como antígeno a B. ovis, amostra 63/290.

\section{Determinação da prevalência}

Para a determinação da prevalência da infecção por Brucella canis, a IDGA foi utilizada como prova de triagem, e a IDGA-ME e a CFT, como testes confirmatórios. Foram consideradas como positivas as amostras que reagiram positivamente nas duas provas confirmatórias.

Estudo de fatores de risco

Os proprietários dos cães que tiveram o sangue colhido responderam a um questionário, que serviu de parâmetro para a análise de fatores de risco. $\mathrm{O}$ questionário abordou dados referentes a idade, sexo, raça, tipo de manejo, abortamentos e viagens.

\section{Análise estatística}

Para o estudo de fatores de risco associados à soropositividade para $B$. canis, foi realizada uma análise univariada através da estimativa pontual e intervalar da odds ratio (OR), executada pelo programa SPSS for Windows 11.0. O resultado foi significativo quando o intervalo de confiança não incluía o valor 1 (um). $O$ teste 
de hipóteses foi realizado por meio do teste exato de Fisher (teste monocaudal) (Zar 1999), e o nível de significância adotado foi de 0,05 .

\section{RESULTADOS}

Dos 410 animais examinados, 39 foram positivos na IDGA, dos quais 15 foram positivos na IDGA-ME e 14 na CFT. Nove animais foram positivos nas duas técnicas confirmatórias. Considerou-se como positivas, para a determinação da prevalência, as amostras que reagiram positivamente no teste de triagem (IDGA) e nas duas provas confirmatórias (IDGAME e CFT). Dessa forma, a prevalência da infecção por Brucella

Quadro 1. Características do grupo amostral da população canina de Santana de Parnaíba, SP, segundo dados obtidos a partir dos questionários aplicados aos proprietários dos cães

\begin{tabular}{lccc}
\hline \multicolumn{2}{c}{ Características } & $\mathrm{n}^{\mathrm{o}}$ & $\%$ \\
\hline \multirow{2}{*}{ Sexo } & Masculino & 238 & 58 \\
& Feminino & 172 & 42 \\
Total & & 410 & 100 \\
Raça & Sem raça definida & 298 & 72,7 \\
& Com raça definida & 112 & 27,3 \\
Total & & 410 & 100 \\
Tipo de manejo & Domiciliar & 266 & 64,9 \\
& Semidomiciliar & 127 & 31 \\
Total & Solto & 17 & 4,1 \\
Viagem & & 410 & 100 \\
& Sim & 26 & 6,3 \\
Total & Não & 384 & 93,7 \\
Abortamento & & 410 & 100 \\
& Sim & 27 & 15,7 \\
Total & Não & 145 & 84,3 \\
Idade & & 172 & 100 \\
& Até 1 ano & 137 & 33 \\
& N 1 ano & 261 & 64 \\
Total & Não sabe & 12 & 3 \\
& & 410 & 100 \\
& & &
\end{tabular}

Quadro 2. Distribuição de positivos e negativos segundo as variáveis estudadas e respectivos valores da odds ratio (OR), intervalo de confiança de $95 \%$ (I.C. 95\%) e a probabilidade de ocorrência ao acaso $(P)$

\begin{tabular}{lccccc}
\hline Variáveis & Descrição & $\begin{array}{c}\text { Posi- } \\
\text { tivos }\end{array}$ & $\begin{array}{c}\text { Nega- } \\
\text { tivos }\end{array}$ & OR $^{\mathrm{a}}(\mathrm{I} . C .95 \%)^{\mathrm{a}}$ & $P^{\mathrm{a}}$ \\
\hline Sexo & Masculino & 5 & 233 & & \\
& Feminino & 4 & 168 & $1,11(0,29-4,19)$ & 0,57 \\
Raça & Com raça definida & 1 & 111 & & \\
& Sem raça definida & 8 & 290 & $3,06(0,38-24,76)$ & 0,24 \\
Viagem & Não & 8 & 376 & & \\
Tipo de & Sim & 1 & 25 & $1,88(0,23-15,63)$ & 0,45 \\
manejo & Domiciliar & 4 & 262 & & \\
Aborta- & Semidomiciliar & 3 & 124 & $1,58(0,35-7,19)$ & 0,41 \\
mentos & Não & 2 & 15 & $8,73(1,48-51,55)$ & 0,04 \\
Idade & Sim & 2 & 143 & $\ldots$ & 0,12 \\
& Até 1 ano & 2 & 135 & & \\
\hline
\end{tabular}

avalores calculados com o programa SPSS for Windows 11.0. canis encontrada no presente trabalho foi de $2,2 \%(9 / 410)$ (I.C. $95 \%=1,01-4,13 \%)$.

No Quadro 1 são apresentadas as características do grupo amostral da população de cães do Município de Santana de Parnaíba, SP.

No Quadro 2 são apresentados os resultados da análise de associação entre as variáveis sexo, raça, tipo de manejo, abortamentos, viagem e idade e a soropositividade para $B$. canis. De acordo com a análise estatística, a única variável associada à ocorrência de soropositivos para $B$. canis foi o tipo de manejo. $O$ manejo do tipo solto apresentou correlação com a soropositividade para $B$. canis, com uma OR de 8,73 (I.C. $95 \%=1,48-51,55$ ) e $\mathrm{p}=0,04$.

\section{DISCUSSÃO}

Utilizando-se a IDGA como teste de triagem e a IDGA-ME e a CFT como testes confirmatórios, a prevalência de anticorpos contra Brucella canis em cães do Município de Santana de Parnaíba, SP, foi de $2,2 \%$ (I.C. 95\% = 1,01-4,13\%), valor esse inferior ao encontrado por Vargas et al. (1996), em Uruguaiana, RS, Megid et al. (1999), em Botucatu, SP, e Nárez et al. (1999), na cidade do México, que obtiveram, respectivamente, $72,7 \%$, $57,1 \%$ e $45 \%$ de positividade. Essa diferença pode ser explicada pelo fato desses estudos terem sido conduzidos em canis comerciais que apresentavam histórico de infertilidade, abortamentos e nascimento de natimortos, o que cria um ambiente extremamente propício para a rápida difusão da infecção (Carmichael \& Greene 1998). No presente estudo, foi examinada uma amostra aleatória de animais pertencentes a diferentes proprietários e distribuídos em diversas áreas do município. Destaca-se, ainda, que a maioria dos animais não apresentava histórico de problemas reprodutivos sugestivos de brucelose por $B$. canis.

Germano et al. (1987) investigaram a prevalência da infecção por B. canis em 352 amostras de soros de cães colhidas durante a campanha de vacinação anti-rábica de Campinas, SP, realizada em agosto de 1981 , e encontraram $5,4 \%$ de positividade, valor este superior ao obtido no presente trabalho. Isso pode ser explicado pela diferença das metodologias utilizadas na amostragem, pois naquele estudo houve a cobertura de apenas $10 \%$ dos postos de vacinação, enquanto o presente estudo abrangeu todos os postos de vacinação. Vale ainda ressaltar que os dois inquéritos empregaram testes sorológicos distintos, o que também pode ter contribuído para a desigualdade dos resultados obtidos (Carmichael \& Greene 1998).

Côrtes et al. (1988) examinaram 3.386 cães errantes capturados pelo programa de controle de reservatórios de zoonoses do Centro de Controle de Zoonoses da Secretaria de Higiene e Saúde do Município de São Paulo, durante o período de 1981 a 1985 , e encontraram $7,5 \%$ de positividade, o que foi superior ao percentual encontrado no presente trabalho. Naquele estudo, foi empregado como teste diagnóstico a imunodifusão em gel de ágar com antígeno produzido pelo Centro Panamericano de Zoonoses (CPZ), a população canina estudada era constituída apenas de cães errantes, e não foram estabelecidos critérios quanto ao tamanho 
amostral, de modo que os animais examinados não estavam distribuídos por toda a extensão geográfica do município, o que poderia justificar as diferenças observadas em relação aos resultados do presente estudo.

A prevalência da brucelose por $B$. canis encontrada no presente trabalho $(2,2 \%)$ foi inferior à observada por Miranda et al. (1986), na província de Formosa, Argentina (15,62\%), em 64 animais examinados. Essa diferença pode ser justificada tanto pelo tamanho da amostra como também por ter sido empregada apenas a imunodifusão em gel de ágar, que pode apresentar resultados falso-positivos (Johnson \& Walker 1992, Carmichael 1998, Carmichal \& Greene 1998, Keid 2001).

A população canina de Santana de Parnaíba, SP, apresentou uma composição heterogênea, e, com o objetivo de avaliar possíveis associações entre a soropositividade para $B$. canis e fatores importantes na epidemiologia da doença, foram analisadas as variáveis: sexo, raça, tipo de manejo, abortamentos, idade e viagem.

Analisando-se o sexo como um possível fator de risco associado à soropositividade para $B$. canis, não foi observada significância estatística, ou seja, não houve predisposição de sexo, estando os machos e as fêmeas igualmente expostos ao risco da infecção. Resultados semelhantes foram encontrados por Hubbert et al. (1980), Germano et al. (1987) e Moraes et al. (2002).

Quando das primeiras descrições de cães infectados por B. canis, os animais da raça Beagle foram considerados como os mais susceptíveis (Moore 1969, Moore \& Gupta 1970, Pickerill \& Carmichael 1972). No entanto, posteriormente a doença passou a ser diagnosticada em cães de raças diversas (Carmichael \& Kenney 1968, Fredrickson \& Barton 1974). No presente trabalho, não foi constatada associação entre a raça e a ocorrência de soropositivos para $B$. canis.

No relativo ao tipo de manejo, observou-se que $1,5 \%$ (4/ 266) dos cães domiciliados, $2,4 \%$ (3/127) dos cães submetidos a um manejo semidomiciliar e $11,8 \%(2 / 17)$ dos animais com acesso irrestrito às ruas (manejo do tipo solto) foram soropositivos. Quando o tipo de manejo foi avaliado como fator de risco, o manejo do tipo solto apresentou significância estatística, com uma OR de 8,73 (I.C. 95\% = 1,48-51,55) e p = 0,04 . O manejo do tipo solto é um evento epidemiológico relevante para a brucelose canina já ressaltado por Moore (1969), Brown et al. (1976), Lovejoy et al. (1976), Galphin (1977), Hubbert et al. (1980) e Larsson et al. (1981), uma vez que animais submetidos a esse regime de manejo podem entrar em contato com outros animais, o que aumenta as chances da infecção (Carmichael \& Greene, 1998). Isso reforça ainda mais a necessidade do controle da população de cães errantes, por meio de castração ou estímulo da posse responsável (Organización Mundial de la Salud/Sociedade Mundial para la Proteccion Animal, WSPA 1990).

Com relação à ocorrência de abortamento, verificou-se que $7,4 \%(2 / 27)$ das fêmeas com histórico de abortamento e $1,4 \%(2 / 145)$ das fêmeas sem histórico de abortamento foram soropositivas para $B$. canis. Em relação a outros estudos, a percentagem de positividade em fêmeas que abortaram observada no presente trabalho foi baixa. Hubbert et al. (1980), em Baton Rouge, Estado da Louisiana, EUA, utilizando a soroaglutinação lenta em tubos em soros tratados pelo 2-mercaptoetanol, encontraram $47,6 \%$ de positivos em 21 cadelas que haviam abortado. Megid et al. (1999), no Brasil, examinando 151 cães da raça Poodle provenientes de quatro canis diferentes, encontraram $50 \%$ de soropositivos entre as fêmeas com história de abortamento. No presente trabalho, não houve associação estatística entre a ocorrência de abortamentos e a soropositividade para $B$. canis, fato este inesperado, pois o abortamento é o principal sinal clínico da brucelose por $B$. canis em cadelas gestantes (Moore 1969, Johnson \& Walker 1992, Carmichael \& Greene 1998). A ausência de associação observada neste estudo pode ser explicada pelo pequeno número de reações positivas entre as fêmeas (4/172), pois certos estudos podem perder poder estatístico quando uma determinada variável ocorre com baixa freqüência (Sasaki et al. 1993), e nesses casos a variável poderá ser considerada como não adequadamente avaliada. Outro ponto a ser levado em consideração é que o relato da ocorrência de abortamento depende muito da observação do proprietário, especialmente quando ocorre no início da gestação, o que pode interferir na avaliação (Mascolli et al. 2002).

No relativo à idade, observou-se que 77,8\% (7/9) dos animais positivos tinham mais de um ano de idade. Resultados similares foram obtidos por Hubbert et al. (1980), Germano et al. (1987) e Moraes et al. (2002), que encontraram uma maior proporção de resultados positivos em animais com idade superior a um ano. Isso pode ser justificado pela maturidade sexual e conseqüente cobertura, bem como pela maior possibilidade de contato com animais infectados em função da idade (Johnson \& Walker 1992, Carmichael \& Greene 1998). Quando a faixa etária foi analisada como possível fator de risco associado à soropositividade para $B$. canis, não foi observada significância estatística, ou seja, animais impúberes e sexualmente maduros estão igualmente expostos ao risco da infecção. Animais impúberes podem adquirir a infecção e se tornarem bacterêmicos, e nesse caso normalmente a manifestação clínica é apenas uma linfadenopatia uni ou bilateral. No entanto, os sinais clínicos reprodutivos só se manifestam após a puberdade (Carmichael \& Kenney 1970, Greene \& George 1984, Johnson \& Walker 1992).

Com relação à análise de viagem como possível fator de risco associado à soropositividade para $B$. canis, não foi verificada significância estatística, ou seja, embora os animais que acompanham seus proprietários durante viagens possam entrar em contato com outros cães, no município estudado esse fato não foi um fator de risco para a doença.

\section{REFERÊNCIAS}

Alves F.A.L., Alves C.J., Azevedo S.S., Andrade J.S.L. \& Santos F.A.S. 1999. Prevalëncia de anticorpos anti-Brucella canis em cães na cidade de Patos, PB. Anais IV Congr. Pernamb. Med. Veterinária, 17-20 ago., Recife, PE, p. 259-260.

Brown J., Blue J.L., Wooley R.E., Dreesen D.W. \& Carmichael L.E. 1976. A serologic survey of a population of Georgia dogs for Brucella canis and an evaluation of the slide agglutination test. J. Am. Vet. Med. Assoc. 169(11):1214-1216. 
Carmichael L.E. 1966. Abortions in 200 Beagles. J. Am. Vet. Med. Assoc. 149(8): 1126.

Carmichael L.E. 1990. Brucella canis, p. 335-350. In: Nielsen K. \& Duncan J.R. (ed.) Animal Brucellosis. CRC Press, Boca Raton, Florida.

Carmichael L.E. 1998. Brucelosis canina causada por B. canis: enfermidade clínica; problemas en inmunodiagnóstico. Revta Med. Vet. 80(2):102-106.

Carmichael L.E. \& Greene C.E. 1998. Canine brucellosis, p. 248-257. In: Greene C.E. (ed.) Infectious Diseases of the Dog and Cat. 2nd ed. W.B. Saunders, Philadelphia.

Carmichael L.E. \& Kenney R.M. 1968. Canine abortion caused by Brucella canis. J. Am. Vet. Med. Assoc. 152(6):605-616.

Carmichael L.E. \& Kenney R.M. 1970. Canine brucellosis: the clinical disease, pathogenesis, and immune response. J. Am. Vet. Med. Assoc. 156(12):17261734.

Côrtes V.A., Oliveira M.C.G. Ito, F.H. \& Vasconcellos S.A. 1988. Reações sorológicas para Brucella canis em cães errantes capturados na proximidade dos parques públicos, reservas florestais e em áreas periféricas do município de São Paulo-Brasil. Revta Fac. Med. Vet. Zootec. Univ. S. Paulo 25(1):101-107.

Fernandes J.C.T., Wald V.B. \& Jobim G.B. 1976/77. Isolamento de Brucella canis do humor aquoso de um cão com lesões oculares. Arq. Fac. Vet. Univ. Fed. Rio Grande do Sul 4(5):109-113.

Fredrickson L.E. \& Barton C.E. 1974. A serologic survey for canine brucellosis in a metropolitan area. J. Am. Vet. Med. Assoc. 165(11):987-989.

Galphin S.P. 1977. A serological survey for Brucella canis in dogs on a military base. J. Am. Vet. Med. Assoc. 171(8):728-729.

Garin-Bastuji G. \& Blasco J.M. 1996. Ovine epididymitis (Brucella ovis), p. 343349. In: Manual of standards for diagnostic tests and vaccines. O.I.E., Paris.

Germano P.M.L., Vasconcellos S.A., Ishizuka M.M., Passos E.C. \& Erbolato E.B. 1987. Prevalência de infecção por Brucella canis em cães da cidade de Campinas-SP, Brasil. Revta Fac. Med. Vet. Zootec. Univ. S. Paulo 24(1):27-34.

Godoy A.M., Peres J.N. \& Barg L. 1977. Isolamento de Brucella canis em Minas Gerais, Brasil. Arqs Esc. Vet. Univ. Fed. Minas Gerais 29(1):35-42.

Gomes M.J.P., Driemeier D., Soares H.C., Bastos C.D., Canto S.P., Brum M., Rossi A.C. \& Corbellini L.G. 1999. Brucella canis: isolamento em um cão com epididimite e orquite - relato de caso. Clínica Vet. 4(18):17-20.

Greene C.E. \& George L.W. 1984. Canine brucellosis, p. 646-662. In: Greene C.E. (ed.) Clinical Microbiology and Infectious Diseases of the Dog and Cat. W.B. Saunders, Philadelphia.

Hartigan P.J. 1997. Human brucellosis: epidemiology and clinical manifestations. Irish Vet. J. 50(3):179-180.

Hubbert N.L., Bech-Nielsen S. \& Barta O. 1980. Canine brucellosis: comparison of clinical manifestations with serologic test results. J. Am. Vet. Med. Assoc. 177(2):168-171.

Johnson C.A. \& Walker R.D. 1992. Clinical signs and diagnosis of Brucella canis infection. Comp. Cont. Educ. Pract. Vet. Small Animal 14(6):763-772.

Keid L.B. 2001. Diagnóstico da brucelose canina por Brucella canis. Correlação entre exames clínicos e laboratoriais: imunodifusão em gel de ágar, imunodifusão em gel de ágar com emprego do 2-mercaptoetanol, cultivo e reação em cadeia pela polimerase. Dissertação de Mestrado em Reprodução Animal, Faculdade de Medicina Veterinária e Zootecnia da Universidade de São Paulo, SP. 96p.

Larsson M.H.M.A. 1979. Estudo epidemiológico da brucelose canina. Tese de Doutorado em Saúde Pública, Faculdade de Saúde Pública da Universidade de São Paulo, SP. 50p.

Larsson M.H.M.A. \& Costa E.O. 1980. Isolation of Brucella canis. Int. J. Zoonoses $7(2): 125-130$

Larsson M.H.M.A., Larsson C.E., Mirandola R.M.S., Yassuda P.H. \& Grutolla G. 1981. Canine brucellosis in São Paulo: serologic survey of kennel and stray dogs. Int. J. Zoonoses 8(1):85-90.

Lovejoy G.S., Carver H.D., Moseley I.K. \& Hicks M. 1976. Serosurvey of dogs for Brucella canis infection in Memphis, Tennessee. Am. J. Pub. Health 66(2):175-176.

Maia G.R., Rossi C.R.S., Abbadia F., Vieira D.K. \& Moraes I.A. 1999. Prevalência da brucelose canina nas cidades do Rio de Janeiro e Niterói, RJ. Revta Bras. Reprod. Animal 23(3):425-427.

Mascolli R., Pinheiro S.R., Vasconcellos S.A., Ferreira F., Morais Z.M., Pinto C.O., Sucupira M.C.A., Dias R.A., Miraglia F., Cortez A., Costa S.S., Tabata R. \& Marcondes A.G. 2002. Inquérito sorológico para leptospirose em cães do Município de Santana de Parnaíba, São Paulo, utilizando a campanha de vacinação anti-rábica do ano de 1999. Arqs Inst. Biol., São Paulo, 69(2):25-32.

Megid J., Brito A.F., Moraes C.C.G., Fava N. \& Agottani J. 1999. Epidemiological assessment of canine brucellosis. Arq. Bras. Med. Vet. Zootec. 51(5):439440 .

Melo S.M.B., Aguiar P.H.P., Nascimento R.M. \& Freire S.M. 1997/98. Avaliação sorológica por imunodifusão em gel de agarose para diagnóstico de Brucella canis em cães no distrito de Monte Gordo, Camaçari, Bahia. Arqs Esc. Med. Vet. Univ. Fed. Bahia 19(1):119-127.

Miranda A.O., Colman O.L.R., Mancebo O.A. \& Monzón C.M. 1986. Deteccion serologica de anticuerpos anti Brucella canis en perros y humanos en el oeste de Formosa. Vet. Arg. 3(22):158-161.

Molnár L., Molnár E. \& Carvalho M. 2001. Capacidade de algumas provas sorológicas no diagnóstico de brucelose canina. Hora Vet. 21(121):45-49.

Moore J.A. 1969. Brucella canis infection in dogs. J. Am. Vet. Med. Assoc. 155(12): 2034-2037.

Moore J.A. \& Gupta B.N. 1970. Epizootiology, diagnosis, and control of Brucella canis. J. Am. Vet. Med. Assoc. 156(12):1737-1742.

Moraes C.C.G., Megid J., Souza L.C. \& Crocci A.J. 2002. Prevalência da brucelose canina na microrregião da serra de Botucatu, São Paulo, Brasil. Arqs Inst. Biológico, São Paulo, 69(2):7-10.

Moraes I.A., Laranja H.F., Vieira D.K., Lopes S., Freaza A., Melo G. \& Penchel V. 2001. Identificação de cães potenciais transmissores de brucelose na Zona Oeste da cidade do Rio de Janeiro. Anais XXVIII Congr. Bras. Med. Veterinária, 11-15 nov., Salvador, BA, p. 163.

Nárez G.M., Cortés E.M., Reynoso B.A., Basilio J.I.M. \& Aparicio E.D. 1999. Seguimiento de un brote de Brucella canis en un criadero de perros en la ciudad de Mexico. Tec. Pec. Mexico 37(3):43-50.

Organización Mundial de la Salud/Sociedade Mundial para la Proteccion Animal (WSPA). Guias para el manejo de la población canina. 1990. Ginebra. $128 \mathrm{p}$.

Pickerill P.A. \& Carmichael L.E. 1972. Canine brucellosis: control programs in commercial kennels and effect on reproduction. J. Am. Vet. Med. Assoc. 160(12):1607-1615.

Reichmann M.L.A.B., Pinto H.B.F. \& Nunes V.F.P. 1999. Vacinação contra a raiva de cães e gatos. Manuais 3, Inst. Pasteur, São Paulo. 32p.

Roxo E., Pinheiro S.R., Brandão M.M., Aguiar J.A.C., Gouvêa G., Piorum M.L. \& Lima M.A.B. 1990. Brucelose canina: relato de uma possível transmissão de Brucella canis ao homem a partir de uma cadela da raça Doberman. Bolm Inform. Cont. Zoon. Urbanas 13(1):47-49.

Sandoval L.A., Ribeiro L.O.C., Amaral L.B.S., Feitosa M.H. \& Bazan J.M. 1976. Incidência da brucelose canina na cidade de São Paulo. Biológico, São Paulo, 42(5-6):128-132.

Sasaki D.M., Pang L., Minette H.P., Wakida C.K., Fujimoto W.J., Manea S.J., Kunioka R. \& Middleton C.R. 1993. Active surveillance and risk factors for leptospirosis in Hawaii. Am. J. Trop. Med. Hygiene 48(1):35-43.

Vargas A.C., Lazzari A., Dutra V. \& Poester F. 1996. Brucelose canina: relato de caso. Ciência Rural, Santa Maria, 26(2):305-308.

Zar J.H. 1999. Biostatistical Analysis. 4th ed. Prentice Hall, Upper Saddle River. $663 \mathrm{p}$.

Wald V.B. \& Fernandes J.C.T. 1976/77. Sorologia da brucelose canina no município de Porto Alegre, RS. Arq. Fac. Vet. Univ. Fed. Rio Grande do Sul 4(5):9295. 